УДК 519.213

\title{
Synthesis of an Algorithm of Space-time Processing Received Satellite Navigation Signal and Spoofing Jamming
}

\author{
Alexander I. Perov* \\ Sergey P. Ippolitov ${ }^{\dagger}$ \\ National Research University "Moscow Power Engineering Institute"
}

Krasnokazarmennaya, 14, Moscow, 111250

Russia

Received 09.01.2017, received in revised form 20.03.2017, accepted 10.08.2017

In the article, the theory of optimal filtering of information processes is used to synthesize an algorithm of space-time processing received satellite navigation signal and spoofing jamming. Equations of the optimal space-time filtering are cited. Statistical analysis results are presented that confirm performance of synthesized algorithm.

Keywords: satellite navigation, spoofing jamming, optimal algorithm, tracking, space-time processing. DOI: 10.17516/1997-1397-2017-10-4-429-442.

\section{Introduction}

Global navigation systems (GLONASS, GPS) are widely used in different technical field of use $[1,2]$. Navigation receivers provide high accuracy for coordinate definitions $(\approx 2 \ldots 3 \mathrm{~m})$. But they are subjected to radio jamming. To protect navigation receivers against jamming there used methods of spatial jammer suppression [3-7] based on using an antenna array and spatial processing algorithms that form a gap at jammer direction in an antenna pattern. Efficiency of these algorithms is increasing when jammer power is increasing relative to navigation signal power. Typical value of such power elevation can be $30 \mathrm{~dB}$ and greater. But apart from powerful barrage jamming there are used spoofing jamming that imitate the true navigation signals arriving from GPS/GLONASS satellites. Required power for these jamming is just only 4-5 dB grater then navigation signal power. Principle its action is following. Parameters of a spoofing jamming are determined in that way that they get into limits of a delay discriminator characteristic and has appropriate Doppler shift. As power of a jamming signal is grater then power of a navigation signal then the receiver will be track a jamming signal. If in the receiver there are used an antenna array it can not efficiently suppress such jammer and correct operation of the receiver will be interrupted $[8,9]$. The matter of this article is synthesis of an optimal algorithm of space-time processing received satellite navigation signal and a spoofing jamming that results reliable navigation signal tracking.

\footnotetext{
*alexp@aha.ru

†ippolitovsp@gmail.om

(c) Siberian Federal University. All rights reserved
} 


\section{Setting synthesis problem}

We have a $m$-elements planar antenna array (AA) with which the coordinate system (CS) OXYZ is connected (Fig. 1). In this SC $l$-th antenna element position is described by vector $\boldsymbol{\rho}_{A_{l}}$. A navigation signal comes at AA from the direction specified by the unit vector $\boldsymbol{e}_{s}$ and a jamming signal comes at AA from the direction specified by the unit vector $\boldsymbol{e}_{j a m}$.

Let's consider that navigation and jamming signals have identical structure and when they are received at the point $\mathrm{O}$ in the OXYZ SC at fixed time $t_{0}$ they have equal delay and Doppler shift values. This situation is typical when there are used aimed spoofing jamming affected on a receiver of GPS/GLONASS navigation signals. In addition it is supposed that there are estimated angles of the vector $\boldsymbol{e}_{j a m}$ using AA and methods such as MUSIC or ESPRIT and others $[10,11]$.

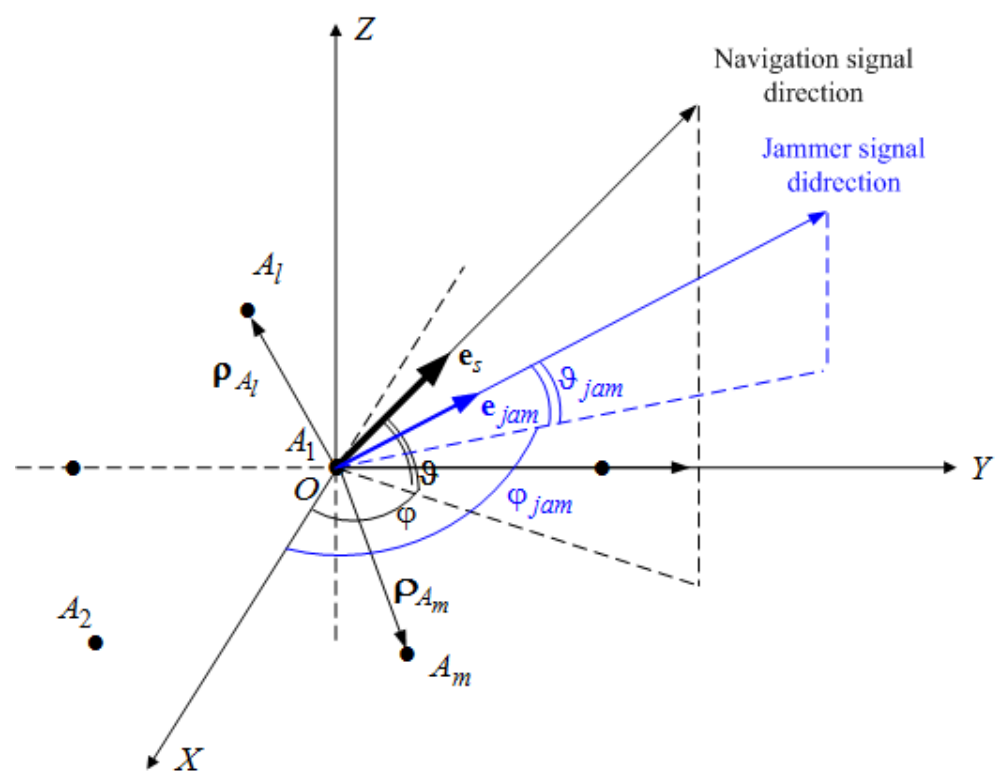

Fig. 1. Geometric scheme of signals reception

At outputs of AA elements at discrete time $t_{k, i}=t_{k}+i T_{d} ; k=1,2, \ldots, i=\overline{1, M}\left(t_{k, M}=t_{k+1}\right.$, $t_{k+1}-t_{k}=T$ is a period of range code modulation) sampling increment $T_{d}$ there are received additive mix of a navigation signal, a jamming signal and discrete white Gaussian noise

$$
\boldsymbol{y}\left(t_{k, i}\right)=\boldsymbol{s}\left(t_{k, i}, \boldsymbol{\lambda}_{k}\right)+\boldsymbol{s}_{j a m}\left(t_{k, i}, \boldsymbol{\lambda}_{j a m ; k}\right)+\boldsymbol{n}\left(t_{k, i}\right),
$$

where

$$
\boldsymbol{s}\left(t_{k, i}, \boldsymbol{\lambda}_{k}\right)=A h_{r c}\left(t_{k, i}-\tau_{k}\right) h_{d i, k} \boldsymbol{s}_{h a r m}\left(t_{k, i}, \varphi_{k, i}\right)
$$

is a navigation signal vector at AA elements that are fitted with vector $\boldsymbol{e}_{s}$ direction, $h_{r c}\left(t_{k, i}\right)$ is range code modulation [12];

$h_{d i, k}$ is digital information transmitted in the navigation signal using BPSK method with symbol duration $\tau_{d i} \geqslant T$ and is a random variable that takes on values \pm 1 ;

$\boldsymbol{s}_{\text {harm }}\left(t_{k, i}, \varphi_{k, i}\right)=\left|\begin{array}{llll}\cos \left(\omega t_{k, i}+\varphi_{k, i}\right) & \cos \left(\omega t_{k, i}+\psi_{2}+\varphi_{k, i}\right) & \ldots & \cos \left(\omega t_{k, i}+\psi_{m}+\varphi_{k, i}\right)\end{array}\right|^{T}$ is a vector of radio frequency harmonic signals in which $\varphi_{k, i}=\varphi_{k}+\omega_{D o p, k} i T_{d}$ is a signal phase that is common for all components at each AA elements; 
element;

$\psi_{l}=\frac{2 \pi \boldsymbol{\rho}_{A_{l}} \boldsymbol{e}_{s}}{\lambda}$ is a phase increment at the $l$-th AA element relative to phase at the first AA

$\lambda_{k}=\left|\tau_{k} \quad \varphi_{k} \quad \omega_{, k}\right|^{T}$ is a vector of estimated parameters;

$$
\boldsymbol{s}_{j a m}\left(t_{k, i}, \boldsymbol{\lambda}_{j a m ; k}\right)=A h_{r c}\left(t_{k, i}-\tau_{j a m ; k}\right) h_{d i, k} \boldsymbol{s}_{h a r m, j a m}\left(t_{k, i}, \varphi_{j a m ; k, i}\right)
$$

is a jamming signal vector at AA elements that are fitted with vector $\boldsymbol{e}_{j a m}$ direction; $h_{r s}\left(t_{k, i}\right)$ is range code modulation the is identical to navigation signal range code (a spoofing jamming );

$$
\begin{aligned}
& \boldsymbol{s}_{\text {harm }, j a m}\left(t_{k, i}, \varphi_{j a m ; k, i}\right)= \\
& =\left|\begin{array}{llll}
\cos \left(\omega t_{k, i}+\varphi_{j a m ; k, i}\right) & \cos \left(\omega t_{k, i}+\psi_{j a m ; 2}+\varphi_{j a m ; k, i}\right) & \ldots & \cos \left(\omega t_{k, i}+\psi_{j a m ; m}+\varphi_{j a m ; k, i}\right)
\end{array}\right|^{T}
\end{aligned}
$$

is a vector of radio frequency harmonic signals in which $\varphi_{j a m ; k, i}=\varphi_{j a m ; k}+\omega_{D o p, j a m ; k} i T_{d}$ is a jamming phase that is common for all components at each AA elements;

$\psi_{j a m ; l}=\frac{2 \pi \boldsymbol{\rho}_{A_{l}} \boldsymbol{e}_{j a m}}{\lambda}$ is a jamming phase increment at the $l$-th AA element relative to phase at the first AA element;

$h_{d i, k}$ is digital information transmitted in the jamming signal using BPSK method with symbol duration $\tau_{d i} \geqslant T$ and that is identical to navigation signal digital information;

$\lambda_{j a m ; k}=\left|\begin{array}{lll}\tau_{j a m ; k} & \varphi_{j a m ; k} & \omega_{D o p, j a m ; k}\end{array}\right|^{T}$ is a vector of estimated jamming parameters; $\boldsymbol{n}\left(t_{k, i}\right)$ is a vector of uncorrelated noses with correlation matrix $M\left[\boldsymbol{n}\left(t_{k, i}\right) \boldsymbol{n}^{T}\left(t_{k, j}\right)\right]=D_{n} \boldsymbol{I} \delta_{i, j}$, where $\mathrm{T}$ denotes hermitian conjugate.

Phase changing model at clocks $t_{k}, k=1,2, \ldots$ is

$$
\begin{gathered}
\varphi_{k}=\varphi_{k-1}+\omega_{D o p, k-1} T, \\
\omega_{D o p, k}=\omega_{D o p, k-1}+\nu_{k-1} T, \\
\nu_{k}=\nu_{k-1}+\xi_{k-1}, \\
\varphi_{j a m ; k}=\varphi_{j a m ; k-1}+\omega_{D o p, j a m ; k-1} T, \\
\omega_{D o p, j a m ; k}=\omega_{D o p, j a m ; k-1}+\nu_{j a m ; k-1} T . \\
\nu_{j a m ; k}=\nu_{j a m ; k-1}+\xi_{j a m ; k-1},
\end{gathered}
$$

where $T=M T_{d} ; \xi_{k-1}, \xi_{j a m ; k-1}$ are vectors of uncorrelated noses with variances $D_{\xi}, D_{\xi_{j a m}}$.

Phase changing models within each time interval $\left[t_{k}, t_{k+1}\right]$ are described by formulas $\varphi_{k, i}=\varphi_{k}+i T_{d} \omega_{D o p, k}, \varphi_{j a m ; k, i}=\varphi_{j a m ; k}+i T_{d} \omega_{D o p, j a m ; k}$.

Delay changing models are $\tau_{k}=\tau_{k-1}-\frac{\omega_{D o p, k-1}}{2 \pi f_{0}} T, \quad \tau_{j a m ; k}=\tau_{j a m ; k-1}-\frac{\omega_{\text {Dop }, j a m ; k-1}}{2 \pi f_{0}} T$, where $f_{0}$ is a carrier frequency. The task is synthesis of an algorithm of optimal filtering $\boldsymbol{\lambda}_{k}$ and $\boldsymbol{\lambda}_{p, k}$ when space-time observations (1).

\section{Synthesis of optimal filtering algorithm}

For synthesis of optimal filtering algorithm let's use optimal filtering theory [13] according which one must consider a posteriori probability density (APD) $p\left(\boldsymbol{\lambda}_{k}, \boldsymbol{\lambda}_{j a m, k}, h_{d i, k} \mid \boldsymbol{Y}_{0}^{k+1}\right)$ where $\boldsymbol{Y}_{0}^{k+1}$ is the observations set received up to time $t_{k+1}$. Using the law of total expectation let's write down

$$
\begin{gathered}
p\left(\boldsymbol{\lambda}_{k}, \boldsymbol{\lambda}_{j a m, k}, h_{d i, k} \mid \boldsymbol{Y}_{0}^{k+1}\right)=p\left(\boldsymbol{\lambda}_{k} \mid \boldsymbol{Y}_{0}^{k+1}, \boldsymbol{\lambda}_{j a m, k}, h_{d i, k}\right) p\left(\boldsymbol{\lambda}_{j a m, k}, h_{d i, k} \mid \mathrm{Y}_{0}^{k+1}\right)= \\
=p\left(\boldsymbol{\lambda}_{j a m, k} \mid \mathrm{Y}_{0}^{k+1}, \boldsymbol{\lambda}_{k}, h_{d i, k}\right) p\left(\boldsymbol{\lambda}_{k}, h_{d i, k} \mid \boldsymbol{Y}_{0}^{k+1}\right) . \\
-431-
\end{gathered}
$$


Further let's consider separately conditional APD $p\left(\boldsymbol{\lambda}_{k} \mid \boldsymbol{Y}_{0}^{k+1}, \boldsymbol{\lambda}_{p, k}, h_{d i, k}\right)$ and $p\left(\boldsymbol{\lambda}_{p, k} \mid \mathrm{Y}_{0}^{k+1}, \boldsymbol{\lambda}_{k}, h_{d i, k}\right)$ with appropriate separately estimating of $\hat{\boldsymbol{\lambda}}_{k}$ on the assumption of known values $\boldsymbol{\lambda}_{j a m, k}$ and similarly estimating of $\hat{\boldsymbol{\lambda}}_{j a m, k}$ on the assumption of known value $\boldsymbol{\lambda}_{k}$. For conditional APD $p\left(\boldsymbol{\lambda}_{k} \mid \mathrm{Y}_{0}^{k+1}, \boldsymbol{\lambda}_{j a m, k}, h_{d i, k}\right)$ let's write down [12]

$$
p\left(\boldsymbol{\lambda}_{k} \mid \boldsymbol{Y}_{0}^{k+1}, \boldsymbol{\lambda}_{j a m, k}, h_{d i, k}\right)=c_{1} p\left(\boldsymbol{\lambda}_{k} \mid \boldsymbol{Y}_{0}^{k}, \boldsymbol{\lambda}_{j a m, k}, h_{d i, k}\right) p\left(\boldsymbol{Y}_{k, 1}^{k, M} \mid \boldsymbol{\lambda}_{k}, \boldsymbol{\lambda}_{j a m, k}, h_{d i, k}\right),
$$

where $\boldsymbol{Y}_{k, 1}^{k, M}=\left\{\boldsymbol{y}\left(t_{k, 1}\right), \boldsymbol{y}\left(t_{k, 2}\right), \ldots, \boldsymbol{y}\left(t_{k, M}\right)\right\}$ is the observations set $(1)$ received at the time interval $\left[t_{k}, t_{k+1}\right] ; c_{1}$ is a normalization constant.

Let's consider conditional probability density of the observations $\boldsymbol{Y}_{k, 1}^{k, M}=\left\{\boldsymbol{y}\left(t_{k, 1}\right), \boldsymbol{y}\left(t_{k, 2}\right), \ldots, \boldsymbol{y}\left(t_{k, M}\right)\right\}$ for fixed values $\boldsymbol{\lambda}_{k}=\left|\varphi_{k} \omega_{\text {Dop }, k} \nu_{k} \tau_{k}\right|^{T}, \quad \boldsymbol{\lambda}_{j a m ; k}=$ $\left|\varphi_{j a m ; k} \omega_{D o p, j a m ; k} \nu_{j a m ; k} \tau_{j a m ; k}\right|^{T}$ and $h_{d i, k}$.

$$
\begin{aligned}
& p\left(\boldsymbol{Y}_{k, 1}^{k, M} \mid \boldsymbol{\lambda}_{k}, \boldsymbol{\lambda}_{j a m, k}, h_{d i, k}\right)= \\
&=c \exp \left[\frac{1}{D_{n}} \sum_{i=1}^{M}\left(\boldsymbol{y}\left(t_{k, i}\right)-\frac{1}{2}\left(\boldsymbol{s}\left(t_{k, i}, \boldsymbol{\lambda}_{k, i}, h_{d i, k}\right)+\boldsymbol{s}_{j a m}\left(t_{k, i}, \boldsymbol{\lambda}_{j a m ; k, i}, h_{d i, k}\right)\right)\right)^{T} \times\right. \\
&\left.\times\left(\boldsymbol{s}\left(t_{k, i}, \boldsymbol{\lambda}_{k, i}, h_{d i, k}\right)+\boldsymbol{s}_{j a m}\left(t_{k, i}, \boldsymbol{\lambda}_{j a m ; k, i}, h_{d i, k}\right)\right)\right],
\end{aligned}
$$

where $\mathrm{c}$ is a normalization constant.

Let's transform (7) taking into account that estimated parameters $\boldsymbol{\lambda}_{k}$ are not energy

$$
\begin{aligned}
& p\left(\boldsymbol{Y}_{k, 1}^{k, M} \mid \boldsymbol{\lambda}_{k}, \boldsymbol{\lambda}_{j a m, k}, h_{d i, k}\right)= \\
& =\tilde{c} \exp \left[\frac{1}{D_{n}} \sum_{i=1}^{M}\left(\boldsymbol{y}\left(t_{k, i}\right)-\boldsymbol{s}_{j a m}\left(t_{k, i}, \boldsymbol{\lambda}_{j a m ; k, i}, h_{d i, k}\right)\right)^{T} \boldsymbol{s}\left(t_{k, i}, \boldsymbol{\lambda}_{k, i}, h_{d i, k}\right)\right]
\end{aligned}
$$

where $\tilde{c}$ is another normalization constant is not depended on estimated parameters $\boldsymbol{\lambda}_{k}$.

The random variable $h_{d i, k}$ takes on values \pm 1 with equal probabilities. Tacking this into account let's average (8) with respect to $h_{d i, k}$

$$
\begin{aligned}
p\left(\mathrm{Y}_{k, 1}^{k, M}\right. & \left.\mid \boldsymbol{\lambda}_{k}, \boldsymbol{\lambda}_{j a m, k}\right)= \\
& =\frac{1}{2}\left(p\left(\boldsymbol{Y}_{k, 1}^{k, M} \mid \boldsymbol{\lambda}_{k}, \boldsymbol{\lambda}_{j a m, k}, h_{d i, k}=1\right)+p\left(\boldsymbol{Y}_{k, 1}^{k, M} \mid \boldsymbol{\lambda}_{k}, \boldsymbol{\lambda}_{j a m, k}, h_{d i, k}=-1\right)\right)= \\
& =\tilde{c} \operatorname{ch}\left[\frac{1}{D_{n}} \sum_{i=1}^{M}\left(\boldsymbol{y}\left(t_{k, i}\right)-\boldsymbol{s}_{j a m}\left(t_{k, i}, \boldsymbol{\lambda}_{j a m ; k, i}, h_{d i, k}=1\right)\right)^{T} \boldsymbol{s}\left(t_{k, i}, \boldsymbol{\lambda}_{k, i}, h_{d i, k}=1\right)\right],
\end{aligned}
$$

where $\operatorname{ch}(x)$ is a hyperbolic cosine function.

Conditional probability density (9) we will use for synthesis of optimal filtering of $\boldsymbol{\lambda}_{k}$ vector on the assumption of known values $\boldsymbol{\lambda}_{j a m, k}$ using following common equations for APD [13]

$$
\begin{gathered}
p\left(\boldsymbol{\lambda}_{k} \mid \boldsymbol{Y}_{0}^{k+1}, \boldsymbol{\lambda}_{j a m, k}\right)=c p\left(\boldsymbol{\lambda}_{k} \mid \boldsymbol{Y}_{0}^{k}, \boldsymbol{\lambda}_{j a m, k}\right) p\left(\boldsymbol{Y}_{k, 1}^{k, M} \mid \boldsymbol{\lambda}_{k}, \boldsymbol{\lambda}_{j a m, k}\right), \\
p\left(\boldsymbol{\lambda}_{k} \mid \boldsymbol{Y}_{0}^{k}, \boldsymbol{\lambda}_{j a m, k}\right)=\int_{-\infty}^{\infty} p\left(\boldsymbol{\lambda}_{k-1} \mid \boldsymbol{Y}_{0}^{k}, \boldsymbol{\lambda}_{j a m, k-1}\right) p\left(\boldsymbol{\lambda}_{k} \mid \boldsymbol{\lambda}_{k-1}\right) d \boldsymbol{\lambda}_{k-1} .
\end{gathered}
$$


It is known that when using Gaussian approximation APD $p\left(\boldsymbol{\lambda}_{k} \mid \boldsymbol{Y}_{0}^{k+1}, \boldsymbol{\lambda}_{j a m, k}\right)$ then for equations (10) there are correspond equations of extended Kalman filter for mathematic expectation $\hat{\lambda}_{k}$ and error variance matrix of this APD. As the vector $\lambda_{k}=\left|\varphi_{k} \omega_{, k} \nu_{k} \tau_{k}\right|^{T}$ includes components $\varphi_{k} \omega_{, k} \nu_{k}$ describing phase changing and the component $\tau_{k}$ describing a signal envelope delay then an optimal filtering system is divided into two parts: a filtering system for state vector $x_{\varphi}=\left|\varphi_{k} \omega_{, k} \nu_{k}\right|^{T}$ that describe a signal phase on the assumption of known value $\tau_{k}$ and a filtering system for a component $\tau_{k}$ on the assumption of known value $\boldsymbol{x}_{\varphi}$. Skipping intermediate calculation let's write down the final optimal filtering algorithms for two pointed systems.

Phase-locked loop (PLL) tracking system for a navigation signal $\boldsymbol{s}\left(t_{k, i}, \boldsymbol{\lambda}_{k}\right)$

$$
\hat{\boldsymbol{x}}_{\varphi, k}=\boldsymbol{F} \hat{\boldsymbol{x}}_{\varphi, k-1}+\boldsymbol{K} u_{d i s, \varphi, k} / S_{d i s, \varphi}, \hat{\boldsymbol{x}}_{k}=\left|\begin{array}{lll}
\hat{\varphi}_{k} & \hat{\omega}_{D o p, k} & \hat{\nu}_{k}
\end{array}\right|^{T}, \mathrm{~F}=\left|\begin{array}{ccc}
1 & T & 0 \\
0 & 1 & T \\
0 & 0 & 1
\end{array}\right|
$$

$$
\begin{aligned}
& u_{d i s, \varphi, k}=\frac{\partial \ln p\left(\boldsymbol{Y}_{k, 1}^{k, M} \mid \boldsymbol{\lambda}_{k}, \boldsymbol{\lambda}_{j a m, k}\right)}{\partial \varphi_{k}}= \\
& =\operatorname{th}\left[\frac{A}{D_{n}} \sum_{i=1}^{M}\left(\boldsymbol{y}\left(t_{k, i}\right)-\boldsymbol{s}_{j a m}\left(t_{k, i}, \boldsymbol{\lambda}_{j a m ; k, i}, h_{d i, k}=1\right)\right)^{T} \boldsymbol{s}_{h a r m}\left(t_{k, i}, \tilde{\varphi}_{k}, \tilde{\omega}_{D o p, k}\right) h_{r c}\left(t_{k, i}-\tilde{\tau}_{k}\right)\right] \times \\
& \times \frac{A}{D_{n}} \sum_{i=1}^{M}\left(\boldsymbol{y}\left(t_{k, i}\right)-\boldsymbol{s}_{p}\left(t_{k, i}, \boldsymbol{\lambda}_{j a m ; k, i}, h_{d i, k}=1\right)\right)^{T} \frac{\partial \boldsymbol{s}_{h a r m}\left(t_{k, i}, \tilde{\varphi}_{k}, \tilde{\omega}_{D o p, k}\right)}{\partial \varphi_{k}} h_{r c}\left(t_{k, i}-\tilde{\tau}_{k}\right),
\end{aligned}
$$

Delay-locked loop (DLL) tracking system for a navigation signal $\boldsymbol{s}\left(t_{k, i}, \boldsymbol{\lambda}_{k}\right)$

$$
\begin{gathered}
\hat{\tau}_{k}=\tilde{\tau}_{k}+K_{\tau} u_{d i s, \tau, k} / S_{d i s, \tau}, \tilde{\tau}_{k}=\hat{\tau}_{k-1}-\frac{\hat{\omega}_{D o p, k-1}}{2 \pi f_{0}} T, \\
u_{d i s, \tau, k}=\frac{\partial \ln p\left(\boldsymbol{Y}_{k, 1}^{k, M} \mid \boldsymbol{\lambda}_{k}, \boldsymbol{\lambda}_{j a m, k}\right)}{\partial \tau_{k}}= \\
=\frac{A}{D_{n}} \sum_{i=1}^{M}\left(\boldsymbol{y}\left(t_{k, i}\right)-\boldsymbol{s}_{j a m}\left(t_{k, i}, \boldsymbol{\lambda}_{j a m ; k, i}\right)\right)^{T} \boldsymbol{s}_{h a r m}\left(t_{k, i}, \tilde{\varphi}_{k}, \tilde{\omega}_{D o p, k}\right) \frac{\partial h_{r c}\left(t_{k, i}-\tilde{\tau}_{k}\right)}{\partial \tau_{k}} .
\end{gathered}
$$

In contrast to known PLL and DLL tracking systems [12] in the synthesized tracking systems there are used phase and delay discriminators in which an influence of jamming signal are compensated. This improves characteristics of navigation signal phase and delay tracking.

As deriving the equations (11)-(14) there where considered conditional APD $p\left(\boldsymbol{\lambda}_{k} \mid \boldsymbol{Y}_{0}^{k+1}, \boldsymbol{\lambda}_{p, k}\right)$ on the assumption of known value $\boldsymbol{\lambda}_{j a m, k}$ then in these equations there are contained $\boldsymbol{\lambda}_{j a m, k}$ as known function.

Let's also note that in the phase discriminator (12) and in the delay discriminator (14) at each time interval $\left[t_{k}, t_{k+1}\right]$ there are jointly processed observations (1) belonged to all AA elements with taking into account there space positions that is there are executed space processing of received signals.

Now let's consider conditional APD $p\left(\boldsymbol{\lambda}_{j a m, k} \mid \boldsymbol{Y}_{0}^{k+1}, \boldsymbol{\lambda}_{k}, h_{d i, k}\right)$ supposing than $\boldsymbol{\lambda}_{k}$ is known.

Repeat for this APD the same deductions and assumptions that had been done above for another APD one can get the optimal filtering algorithms for the vector $\boldsymbol{\lambda}_{p, k}$ of a gamming signal 
$\boldsymbol{s}_{j a m}\left(t_{k, i}, \boldsymbol{\lambda}_{j a m ; k, i}\right)$ that we write down in final form PLL tracking system for a gamming signal $\boldsymbol{s}_{j a m}\left(t_{k, i}, \boldsymbol{\lambda}_{j a m ; k, i}\right)$

$$
\begin{aligned}
& \hat{\boldsymbol{x}}_{\varphi_{j a m}, k}=\boldsymbol{F} \hat{\boldsymbol{x}}_{\varphi_{j a m}, k-1}+\boldsymbol{K} u_{d i s, \varphi_{j a m}, k} / S_{d i s, \varphi_{j a m}},
\end{aligned}
$$

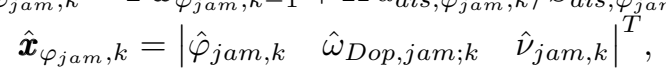

$$
\begin{aligned}
& u_{d i s, \varphi_{j a m}, k}=\frac{\partial \ln p\left(\boldsymbol{Y}_{k, 1}^{k, M} \mid \boldsymbol{\lambda}_{k}, \boldsymbol{\lambda}_{j a m, k}\right)}{\partial \varphi_{j a m ; k}}=\operatorname{th}\left[\frac{A_{j a m}}{D_{n}} \sum_{i=1}^{M}\left(\boldsymbol{y}\left(t_{k, i}\right)-\boldsymbol{s}\left(t_{k, i}, \boldsymbol{\lambda}_{k, i}, h_{d i, k}=1\right)\right)^{T} \times\right. \\
& \left.\times \boldsymbol{s}_{h a r m, j a m}\left(t_{k, i}, \tilde{\varphi}_{j a m ; k}, \tilde{\omega}_{D o p, j a m ; k}\right) h_{r c}\left(t_{k, i}-\tilde{\tau}_{j a m ; k}\right)\right] \times \\
& \times \frac{A_{j a m}}{D_{n}} \sum_{i=1}^{M}\left(\boldsymbol{y}\left(t_{k, i}\right)-\boldsymbol{s}\left(t_{k, i}, \boldsymbol{\lambda}_{k, i}, h_{d i, k}=1\right)\right)^{T} \times \\
& \times \frac{\partial \boldsymbol{s}_{h a r m, j a m}\left(t_{k, i}, \tilde{\varphi}_{j a m ; k}, \tilde{\omega}_{D o p, j a m ; k}\right)}{\partial \varphi_{j a m ; k}} h_{r c}\left(t_{k, i}-\tilde{\tau}_{j a m ; k}\right) .
\end{aligned}
$$

DLL tracking system for a jamming signal $\boldsymbol{s}_{j a m}\left(t_{k, i}, \boldsymbol{\lambda}_{j a m ; k, i}\right)$

$$
\begin{gathered}
\hat{\tau}_{j a m, k}=\tilde{\tau}_{j a m, k}+K_{\tau} u_{d i s, \tau_{j a m}, k} / S_{d i s, \tau_{j a m}}, \tilde{\tau}_{j a m, k}=\hat{\tau}_{j a m, k-1}-\frac{\hat{\omega}_{\text {Dop }, j a m ; k-1}}{2 \pi f_{0}} T, \\
u_{d i s, \tau_{j a m}, k}=\frac{\partial \ln p\left(\boldsymbol{Y}_{k, 1}^{k, M} \mid \boldsymbol{\lambda}_{k}, \boldsymbol{\lambda}_{j a m, k}\right)}{\partial \tau_{j a m, k}}= \\
=\frac{A_{j a m}}{D_{n}} \sum_{i=1}^{M}\left(\boldsymbol{y}\left(t_{k, i}\right)-\boldsymbol{s}\left(t_{k, i}, \boldsymbol{\lambda}_{1 k, i}\right)\right)^{T} \boldsymbol{s}_{h a r m, j a m}\left(t_{k, i}, \tilde{\varphi}_{j a m ; k}, \tilde{\omega}_{D o p, j a m ; k}\right) \frac{\partial h_{r c}\left(t_{k, i}-\tilde{\tau}_{j a m ; k}\right)}{\partial \tau_{j a m ; k}},
\end{gathered}
$$

As deriving the equations (15)-(18) there where considered conditional APD $p\left(\boldsymbol{\lambda}_{, k} \mid \boldsymbol{Y}_{0}^{k+1}, \boldsymbol{\lambda}_{k}\right)$ on the assumption of known value $\boldsymbol{\lambda}_{k}$ then in these equations there are contained $\boldsymbol{\lambda}_{k}$ as known function. On the next step in the synthesis of optimal filtering algorithm we will use sliding filtering method according which in the discriminators (12), (14) it is necessary to use extrapolated estimate $\tilde{\boldsymbol{\lambda}}_{j a m, k}$ formed in the tracking systems (15)-(18) instead of true value $\boldsymbol{\lambda}_{j a m, k}$. Similar in the discriminators (16), (18) it is necessary to use extrapolated estimate $\tilde{\lambda}_{k}$ formed in the tracking systems (11)-(14) instead of true value $\boldsymbol{\lambda}_{k}$.

\section{Analysis of synthesized optimal space-time filtering algorithms}

Analysis of synthesized optimal space-time filtering algorithms in common form is rather cumbersome and not enough visual. So let's do such analysis for 2 elements AA and receiving signals in one plane (Fig. 2).

The antenna element $A_{1}$ we consider as reference and vectors of radio frequency harmonic signals (2) и (3) let's write down in the form

$$
\begin{aligned}
& \boldsymbol{s}_{\text {harm }}\left(t_{k, i}, \varphi_{k, i}\right)=\left|\cos \left(\omega t_{k, i}+\varphi_{k, i}\right) \quad \cos \left(\omega t_{k, i}-\psi \cos (\vartheta)+\varphi_{k, i}\right)\right|^{T}, \\
& \boldsymbol{s}_{\text {harm }, j a m}\left(t_{k, i}, \varphi_{p ; k, i}\right)=\left|\cos \left(\omega t_{k, i}+\varphi_{j a m ; k, i}\right) \cos \left(\omega t_{k, i}-\psi \cos \left(\vartheta_{j a m}\right)+\varphi_{j a m ; k, i}\right)\right|^{T} \text {, }
\end{aligned}
$$


where $\psi=2 \pi d / \lambda$.

From $(12),(14),(16),(18)$ it is followed that discriminators of synthesized system are constructed using correlators of different types. For instance, the phase discriminator (12) of the PLL tracking system for $\boldsymbol{s}\left(t_{k, i}, \boldsymbol{\lambda}_{k}\right)$ signal use the following correlators

$$
\begin{aligned}
& I_{k+1, A_{1}}=\sum_{i=1}^{M}\left(y_{A_{1}}\left(t_{k, i}\right)-A_{j a m} h_{r c}\left(t_{k, i}-\tilde{\tau}_{j a m ; k}\right) \cos \left(\omega t_{k, i}+\tilde{\varphi}_{j a m ; k, i}\right)\right) \times \\
& \times \cos \left(\omega t_{k, i}+\tilde{\varphi}_{k, i}\right) h_{r c}\left(t_{k, i}-\tilde{\tau}_{k}\right) T_{d}, \\
& I_{k+1, A_{2}}=\sum_{i=1}^{M}\left(y_{A_{2}}\left(t_{k, i}\right)-A_{j a m} h_{r c}\left(t_{k, i}-\tilde{\tau}_{j a m ; k}\right) \cos \left(\omega t_{k, i}-\psi \cos \left(\vartheta_{j a m}\right)+\tilde{\varphi}_{j a m ; k, i}\right)\right) \times \\
& \times \cos \left(\omega t_{k, i}-\psi \cos (\vartheta)+\tilde{\varphi}_{k, i}\right) h_{r c}\left(t_{k, i}-\tilde{\tau}_{k}\right) T_{d}, \\
& Q_{k+1, A_{1}}=\sum_{i=1}^{M}\left(y_{A_{1}}\left(t_{k, i}\right)-A_{j a m} h_{r c}\left(t_{k, i}-\tilde{\tau}_{j a m ; k}\right) \cos \left(\omega t_{k, i}+\tilde{\varphi}_{j a m ; k, i}\right)\right) \times \\
& \times \sin \left(\omega t_{k, i}+\tilde{\varphi}_{k, i}\right) h_{r c}\left(t_{k, i}-\tilde{\tau}_{k}\right) T_{d}, \\
& Q_{k+1, A_{2}}=\sum_{i=1}^{M}\left(y_{A_{2}}\left(t_{k, i}\right)-A_{j a m} h_{r c}\left(t_{k, i}-\tilde{\tau}_{j a m ; k}\right) \cos \left(\omega t_{k, i}-\psi \cos \left(\vartheta_{j a m}\right)+\tilde{\varphi}_{j a m ; k, i}\right)\right) \times \\
& \times \sin \left(\omega t_{k, i}-\psi \cos (\vartheta)+\tilde{\varphi}_{k, i}\right) h_{r c}\left(t_{k, i}-\tilde{\tau}_{k}\right) T_{d} .
\end{aligned}
$$

These components are Gaussian random variables that we presenting the form

$$
\begin{aligned}
I_{k+1, A_{1}} & =\bar{I}_{k+1, A_{1}}+I_{f l ; k+1, A_{1}}, & I_{k+1, A_{2}} & =\bar{I}_{k+1, A_{2}}+I_{f l ; k+1, A_{2}}, \\
Q_{k+1, A_{1}} & =\bar{Q}_{k+1, A_{1}}+Q_{f l ; k+1, A_{1}}, & Q_{k+1, A_{2}} & =\bar{Q}_{k+1, A_{2}}+Q_{f l ; k+1, A_{2}},
\end{aligned}
$$

where $\bar{I}_{k+1, A_{1}}, \bar{I}_{k+1, A_{2}}, \bar{Q}_{k+1, A_{1}}, \bar{Q}_{k+1, A_{2}}$ are mathematical expectations of these random variables; $I_{f l ; k+1, A_{1}}, I_{f l ; k+1, A_{2}}, Q_{f l ; k+1, A_{1}}, Q_{f l ; k+1, A_{2}}$ are centered fluctuation parts of these random variables with Gaussian probability densities.

Calculating mathematical expectations of random variables (20) one can get

$$
\begin{gathered}
\bar{I}_{k+1, A_{1}}=h_{d i, k}\left[\frac{A T}{2} \rho\left(\varepsilon_{\tau, k}\right) \cos \left(\varepsilon_{\varphi, k}+\varepsilon_{\omega, k} T / 2\right) \operatorname{sinc}\left(\varepsilon_{\omega, k} T / 2\right)+\right. \\
+\frac{A_{j a m} T}{2} \rho\left(\varepsilon_{\tau, j a m / s ; k}\right) \cos \left(\varepsilon_{\varphi, j a m / s ; k}+\varepsilon_{\omega, j a m / s ; k} T / 2\right) \operatorname{sinc}\left(\varepsilon_{\omega, j a m / s ; k} T / 2\right)- \\
\left.-\frac{A_{j a m} T}{2} \rho\left(\tilde{\varepsilon}_{\tau, j a m / s ; k}\right) \cos \left(\tilde{\varepsilon}_{\varphi, j a m / s ; k}+\tilde{\varepsilon}_{\omega, j a m / s ; k} T / 2\right) \operatorname{sinc}\left(\tilde{\varepsilon}_{\omega, j a m / s ; k} T / 2\right)\right], \\
\bar{Q}_{k+1, A_{1}}=h_{d i, k}\left[\frac{-A T}{2} \rho\left(\varepsilon_{\tau, k}\right) \sin \left(\varepsilon_{\varphi, k}+\varepsilon_{\omega, k} T / 2\right) \operatorname{sinc}\left(\varepsilon_{\omega, k} T / 2\right)-\right. \\
\left.+\frac{A_{j a m} T}{2} \rho\left(\tilde{\varepsilon}_{\tau, j a m / s ; k}\right) \sin \left(\tilde{\varepsilon}_{\varphi, j a m / s ; k}+\tilde{\varepsilon}_{\omega, j a m / s ; k} T / 2\right) \operatorname{sinc}\left(\tilde{\varepsilon}_{\omega, j a m / s ; k} T / 2\right)\right], \\
+\frac{A_{j a m} T}{2} \rho\left(\varepsilon_{\tau, j a m / s ; k}\right) \cos \left(\varepsilon_{\varphi, j a m / s ; k}+\varepsilon_{\vartheta, j a m / s ; k}+\varepsilon_{\omega, j a m / s ; k} T / 2\right) \operatorname{sinc}\left(\varepsilon_{\omega, j a m / s ; k} T / 2\right)- \\
-\frac{A T}{2} \rho\left(\varepsilon_{\tau, k}\right) \cos \left(\varepsilon_{\varphi, k}+\varepsilon_{\omega, k} T / 2\right) \operatorname{sinc}\left(\varepsilon_{\omega, k} T / 2\right)+ \\
\left.\rho\left(\tilde{\varepsilon}_{\tau, j a m / s ; k}\right) \cos \left(\tilde{\varepsilon}_{\varphi, j a m / s ; k}+\varepsilon_{\vartheta, j a m / s ; k}+\tilde{\varepsilon}_{\omega, j a m / s ; k} T / 2\right) \operatorname{sinc}\left(\tilde{\varepsilon}_{\omega, j a m / s ; k} T / 2\right)\right] \\
\bar{Q}_{k+1, A_{2}}=h_{d i, k}\left[\frac{-A T}{2} \rho\left(\varepsilon_{\tau, k}\right) \sin \left(\varepsilon_{\varphi, k}+\varepsilon_{\omega, k} T / 2\right) \operatorname{sinc}\left(\varepsilon_{\omega, k} T / 2\right)-\right. \\
-435-
\end{gathered}
$$




$$
\begin{aligned}
& -\frac{A_{j a m} T}{2} \rho\left(\varepsilon_{\tau, j a m / s ; k}\right) \sin \left(\varepsilon_{\varphi, j a m / s ; k}+\varepsilon_{\vartheta, j a m / s ; k}+\varepsilon_{\omega, j a m / s ; k} T / 2\right) \operatorname{sinc}\left(\varepsilon_{\omega, j a m / s ; k} T / 2\right)+ \\
+ & \left.\frac{A_{j a m} T}{2} \rho\left(\tilde{\varepsilon}_{\tau, j a m / s ; k}\right) \sin \left(\tilde{\varepsilon}_{\varphi, j a m / s ; k}+\varepsilon_{\vartheta, j a m / s ; k}+\tilde{\varepsilon}_{\omega, j a m / s ; k} T / 2\right) \operatorname{sinc}\left(\tilde{\varepsilon}_{\omega, j a m / s ; k} T / 2\right)\right],
\end{aligned}
$$

where
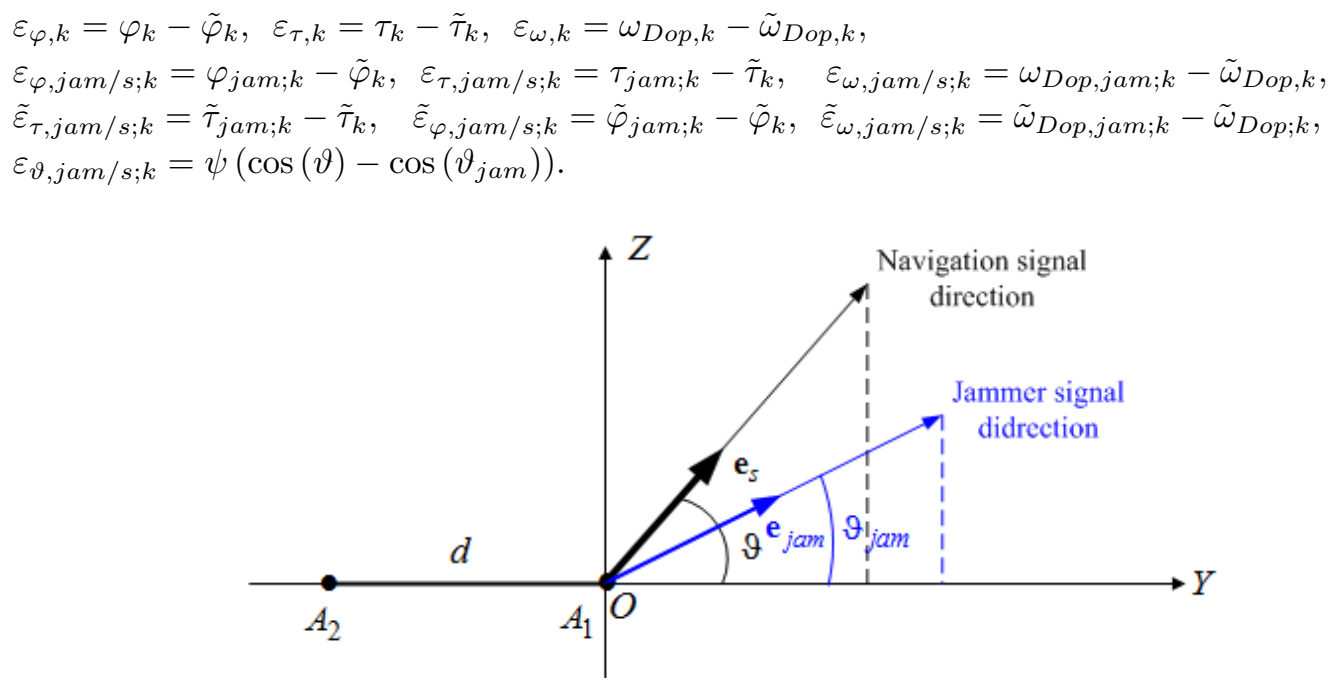

Fig. 2. Geometric scheme of signals reception in the plane

Variances of fluctuation parts of random variables (20) are identical and are defined by the formula

$$
D_{I / Q, f l}=\frac{N_{0} T}{4},
$$

The same formulas can be derived for correlators of phase discriminator (16) that enter into the filtering algorithm for the jamming signal $\boldsymbol{s}_{j a m}\left(t_{k, i}, \boldsymbol{\lambda}_{j a m ; k, i}\right)$.

Let's now consider statistical characteristics of the phase discriminator (12). For typical navigation signal power $\left(P_{s}=-158 d B W\right)$ and integration time in correlators $T \geqslant 1 \mathrm{~ms}$ it can be admitted the following approximation [13]

That is, the function th $\left[\frac{A}{D_{n} T_{d}}\left(I_{k+1, A_{1}}+I_{k+1, A_{2}}\right)\right] \approx h_{d i, k}$ forms an estimate of the digital information symbol. Whereas this assumption let's write down the phase discriminator algorithm (12) in the form

$$
\tilde{u}_{d i s, \varphi, k}=-h_{d i, k}\left(Q_{k+1, A_{1}}+Q_{k+1, A_{2}}\right)=-\left(\tilde{Q}_{k+1, A_{1}}+\tilde{Q}_{k+1, A_{2}}\right),
$$

where $\tilde{Q}_{k+1, A_{1}}=Q_{k+1, A_{1}} / h_{d i, k}, \quad \tilde{Q}_{k+1, A_{2}}=Q_{k+1, A_{2}} / h_{d i, k}$ are Gaussian random variables described by formulas (20) divided on $h_{d i, k}$ (that is by formulas that is in the square bracket) and with equal variances (22).

Let's write down the formula for mathematical expectation of (23)

$$
\begin{aligned}
& \overline{\tilde{u}}_{d i s, \varphi, k}=-\left(\bar{Q}_{k+1, A_{1}}+\bar{Q}_{k+1, A_{2}}\right)= \\
&=A T\left[\rho\left(\varepsilon_{\tau, k}\right) \sin \left(\varepsilon_{\varphi, k}+\varepsilon_{\omega, k} T / 2\right) \operatorname{sinc}\left(\varepsilon_{\omega, k} T / 2\right)+\frac{A_{j a m}}{A} \rho\left(\varepsilon_{\tau, j a m / s ; k}\right) \times\right. \\
& \times \sin \left(\varepsilon_{\varphi, j a m / s ; k}+\varepsilon_{\vartheta, j a m / s ; k} / 2+\varepsilon_{\omega, j a m / s ; k} T / 2\right) \times \cos \left(\varepsilon_{\vartheta, j a m / s ; k} / 2\right) \operatorname{sinc}\left(\varepsilon_{\omega, j a m / s ; k} T / 2\right)-
\end{aligned}
$$




$$
\begin{aligned}
-\frac{A_{j a m}}{A} \rho\left(\tilde{\varepsilon}_{\tau, j a m / s ; k}\right) \sin \left(\tilde{\varepsilon}_{\varphi, j a m / s ; k}+\varepsilon_{\vartheta, j a m / s ; k} / 2+\tilde{\varepsilon}_{\omega, j a m / s ; k} T / 2\right) \times \\
\left.\times \cos \left(\varepsilon_{\vartheta, j a m / s ; k} / 2\right) \operatorname{sinc}\left(\tilde{\varepsilon}_{\omega, j a m / s ; k} T / 2\right)\right] .
\end{aligned}
$$

Variances of fluctuation parts at the output of the discriminator (24) is defined by the formula (adjusted for lack of correlation $Q_{f l ; k+1, A_{1}}$ and $Q_{f l ; k+1, A_{2}}$ )

$$
D_{f l ; d i s, \varphi}=\frac{N_{0} T}{2}
$$

The same way one can get formulas for the jamming signal phase discriminator which we will write down in the final form

$$
\begin{aligned}
\overline{\tilde{u}}_{d i s, \varphi_{j a m}, k}= & A_{j a m} T\left[\rho\left(\varepsilon_{\tau, j a m ; k}\right) \sin \left(\varepsilon_{\varphi, j a m ; k}+\varepsilon_{\omega, j a m ; k} T / 2\right) \operatorname{sinc}\left(\varepsilon_{\omega, j a m ; k} T / 2\right)+\right. \\
+ & \frac{A}{A_{j a m}} \rho\left(\varepsilon_{\tau, s / j a m ; k}\right) \sin \left(\varepsilon_{\varphi, s / j a m ; k}+\varepsilon_{\omega, s / j a m ; k} T / 2-\varepsilon_{\vartheta, j a m / s ; k} / 2\right) \times \\
& \times \cos \left(\varepsilon_{\vartheta, j a m / s ; k} / 2\right) \operatorname{sinc}\left(\varepsilon_{\omega, s / j a m ; k} T / 2\right)-\frac{A}{A_{j a m}} \rho\left(\tilde{\varepsilon}_{\tau, j a m / s ; k}\right) \times \\
\times & \sin \left(\tilde{\varepsilon}_{\varphi, j a m / s ; k}+\varepsilon_{\vartheta, j a m / s ; k} / 2+\tilde{\varepsilon}_{\omega, j a m / s ; k} T / 2\right) \cos \left(\varepsilon_{\vartheta, j a m / s ; k} / 2\right) \times \\
& \left.\times \operatorname{sinc}\left(\tilde{\varepsilon}_{\omega, j a m / s ; k} T / 2\right)\right],
\end{aligned}
$$

where $\varepsilon_{\varphi, j a m ; k}=\varphi_{j a m ; k}-\tilde{\varphi}_{j a m ; k} ., \varepsilon_{\tau, j a m ; k}=\tau_{j a m ; k}-\tilde{\tau}_{j a m ; k}, \varepsilon_{\omega, j a m ; k}=\omega_{D o p, j a m ; k}-\tilde{\omega}_{D o p, j a m ; k}$, $\varepsilon_{\varphi, s / j a m ; k}=\varphi_{k}-\tilde{\varphi}_{j a m ; k}, \quad \varepsilon_{\tau, s / j a m ; k}=\tau_{k}-\tilde{\tau}_{j a m ; k}, \quad \varepsilon_{\omega, s / j a m ; k}=\omega_{D o p, k}-\tilde{\omega}_{D o p, j a m ; k}$.

Variance of fluctuation parts at the output of the discriminator (26) is defined by the formula (25). Let's consider the delay discriminator (14). Changing calculation a derivative on delay with finite differences let's write down

$$
\frac{\partial h_{r c}\left(t_{k, i}-\tilde{\tau}_{k}\right)}{\partial \tau_{k}} \approx \frac{h_{r c}\left(t_{k, i}-\left(\tilde{\tau}_{k}+\Delta \tau / 2\right)\right)-h_{r c}\left(t_{k, i}-\left(\tilde{\tau}_{k}-\Delta \tau / 2\right)\right)}{\Delta \tau},
$$

where $\Delta \tau$ is a delay difference.

Taking into account (27) further we will consider delay discriminator of the form

$$
\begin{aligned}
\tilde{u}_{d i s, \tau, k}=\sum_{i=1}^{M}\left(\boldsymbol{y}\left(t_{k, i}\right)-\boldsymbol{s}_{j a m}(\right. & \left.\left.t_{k, i}, \boldsymbol{\lambda}_{j a m ; k, i}\right)\right)^{T} \boldsymbol{s}_{h a r m}\left(t_{k, i}, \tilde{\varphi}_{k}, \tilde{\omega}_{j a m, k}\right) \times \\
\times & \frac{h_{r c}\left(t_{k, i}-\left(\tilde{\tau}_{k}+\Delta \tau / 2\right)\right)-h_{r c}\left(t_{k, i}-\left(\tilde{\tau}_{k}-\Delta \tau / 2\right)\right)}{\Delta \tau} T_{d} .
\end{aligned}
$$

This delay discriminator includes correlators in which a range code replicas $h_{r c}\left(t_{k, i}-\tilde{\tau}_{k}\right)$. are displaced by $\Delta \tau / 2$ "early" and "late". If to perform calculation of such correlators statistical characteristics using described above method one can get that in this case in formulas for mathematical expectations it is necessary to use $\rho\left(\varepsilon_{\tau}-\Delta \tau / 2\right)$ instead of $\rho\left(\varepsilon_{\tau}\right)$ for correlators with $h_{r c}\left(t_{k, i}-\left(\tilde{\tau}_{k}+\Delta \tau / 2\right)\right)$ and to use $\rho\left(\varepsilon_{\tau}+\Delta \tau / 2\right)$ for correlators with $h_{r c}\left(t_{k, i}-\left(\tilde{\tau}_{k}-\Delta \tau / 2\right)\right)$. Variances of fluctuation parts at the output of early and late correlators are also described by the formula (22).

Let's write down the final formula for mathematical expectation of the random variable (28)

$$
\overline{\tilde{u}}_{d i s, \tau, k}=\frac{A T}{\Delta \tau}\left[\left(\rho\left(\varepsilon_{\tau, k}-\Delta \tau / 2\right)-\rho\left(\varepsilon_{\tau, k}+\Delta \tau / 2\right)\right) \cos \left(\varepsilon_{\varphi, k}+\varepsilon_{\omega, k} T / 2\right) \operatorname{sinc}\left(\varepsilon_{\omega, k} T / 2\right)+\right.
$$




$$
\begin{aligned}
& +\frac{A_{\text {jam }}}{A}\left(\rho\left(\varepsilon_{\tau, j a m / s ; k}-\Delta \tau / 2\right)-\rho\left(\varepsilon_{\tau, j a m / s ; k}+\Delta \tau / 2\right)\right) \times \\
& \times \cos \left(\varepsilon_{\varphi, j a m / s ; k}+\varepsilon_{\omega, j a m / s ; k} T / 2+\varepsilon_{\vartheta, j a m / s ; k} / 2\right) \times \cos \left(\varepsilon_{\vartheta, j a m / s ; k} / 2\right) \operatorname{sinc}\left(\varepsilon_{\omega, j a m / s ; k} T / 2\right)- \\
& -\frac{A_{j a m}}{A}\left(\rho\left(\tilde{\varepsilon}_{\tau, j a m / s ; k}-\Delta \tau / 2\right)-\rho\left(\tilde{\varepsilon}_{\tau, j a m / s ; k}+\Delta \tau / 2\right)\right) \times \\
& \times \cos \left(\tilde{\varepsilon}_{\varphi, j a m / s ; k}+\tilde{\varepsilon}_{\omega, j a m / s ; k} T / 2+\varepsilon_{\vartheta, j a m / s ; k} / 2\right) \times \\
& \left.\times \cos \left(\varepsilon_{\vartheta, j a m / s ; k} / 2\right) \operatorname{sinc}\left(\tilde{\varepsilon}_{\omega, j a m / s ; k} T / 2\right)\right] .
\end{aligned}
$$

Variance of the random variable (28) is defined by the formula

$$
D_{f l ; d i s, \tau}=\frac{N_{0} T}{\Delta \tau^{2}} .
$$

For mathematical expectation of a reading of a jamming signal delay discriminator one can get the following formula

$$
\begin{gathered}
\overline{\tilde{u}}_{d i s, \tau_{j a m}, k}=\frac{A_{j a m} T}{\Delta \tau}\left[\left(\rho\left(\varepsilon_{\tau_{j a m}, k}-\Delta \tau / 2\right)-\rho\left(\varepsilon_{\tau_{j a m}, k}+\Delta \tau / 2\right)\right) \times\right. \\
\times \cos \left(\varepsilon_{\varphi, j a m ; k}+\varepsilon_{\omega, j a m ; k} T / 2\right) \operatorname{sinc}\left(\varepsilon_{\omega, k} T / 2\right) \\
+\frac{A_{j a m}}{A}\left(\rho\left(\varepsilon_{\tau, s / j a m ; k}-\Delta \tau / 2\right)-\rho\left(\varepsilon_{\tau, s / j a m ; k}+\Delta \tau / 2\right)\right) \times \\
\times \cos \left(\varepsilon_{\varphi, s / j a m ; k}-\varepsilon_{\vartheta, j a m / s ; k} / 2+\varepsilon_{\omega, s / j a m ; k} T / 2\right) \cos \left(\varepsilon_{\vartheta, j a m / s ; k} / 2\right) \operatorname{sinc}\left(\varepsilon_{\omega, s / j a m ; k} T / 2\right)- \\
-\frac{A_{j a m}}{A}\left(\rho\left(\tilde{\varepsilon}_{\tau, j a m / s ; k}-\Delta \tau / 2\right)-\rho\left(\tilde{\varepsilon}_{\tau, j a m / s ; k}+\Delta \tau / 2\right)\right) \times \\
\times \cos \left(\tilde{\varepsilon}_{\varphi, j a m / s ; k}+\tilde{\varepsilon}_{\omega, j a m / s ; k} T / 2+\varepsilon_{\vartheta, j a m / s ; k} / 2\right) \times \\
\left.\times \cos \left(\varepsilon_{\vartheta, j a m / s ; k} / 2\right) \operatorname{sinc}\left(\tilde{\varepsilon}_{\omega, j a m / s ; k} T / 2\right)\right] .
\end{gathered}
$$

Variance of fluctuation parts at the output of a jamming signal delay discriminator is defined by the formula (30).

Through substitution formulas (24), (26), (29), (31) into equations (11), (13), (15), (17) we get complex filtering system equations that describe regular processes transformation in the system.

Synthesized optimal space-time filtering algorithm is simulated using following conditions:

a jamming signal amplitude is 5 time grater then a navigation signal amplitude;

initially delays and Doppler shifts of the jamming signal and the navigation signal are agreed within measurement inaccuracy (aimed spoofing jamming );

in the coarse of simulation delay and Doppler shift laws of variation for the jamming signal and the navigation signal are different;

PLL bandwidth is $\Delta f_{P L L}=20 \mathrm{~Hz}$ (for jamming and navigation signals tracking);

DLL bandwidth is $\Delta f_{P L L}=2 \mathrm{~Hz}$ (for jamming and navigation signals tracking); signal type is GPS C/A code;

correlator integration time $T=1 \mathrm{~ms}$.

Fig. 3 shows a realization of a navigation signal phase tracking error, Fig. 4 shows a realization of a navigation signal delay tracking error, Fig. 5 shows a realization of a jamming signal phase tracking error, Fig. 6 shows a realizations of a jamming signal delay tracking error.

Fig. 7 shows a realization delay difference between navigation and jamming signals delays during simulation. 


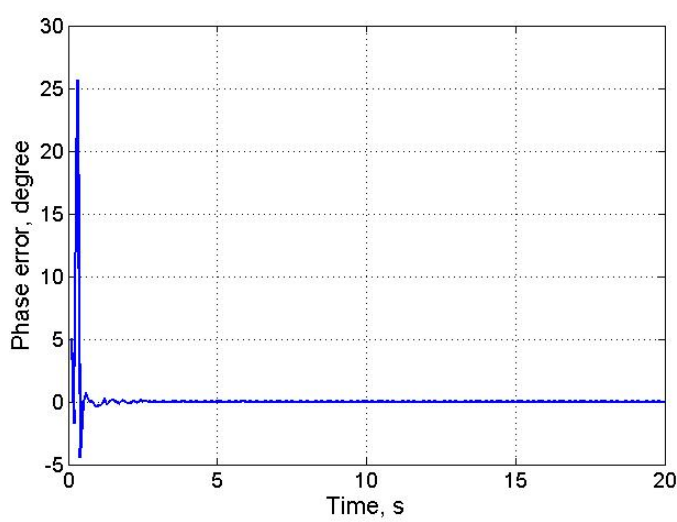

Fig. 3. A realization of a navigation signal phase tracking error

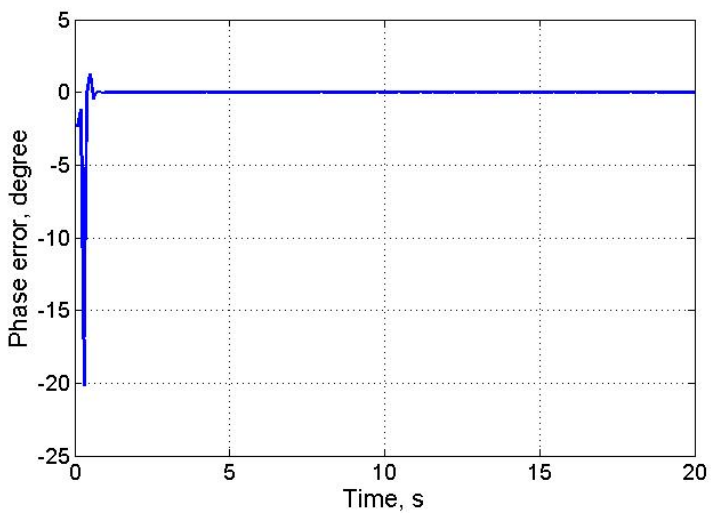

Fig. 5. A realization of a jamming signal phase tracking error

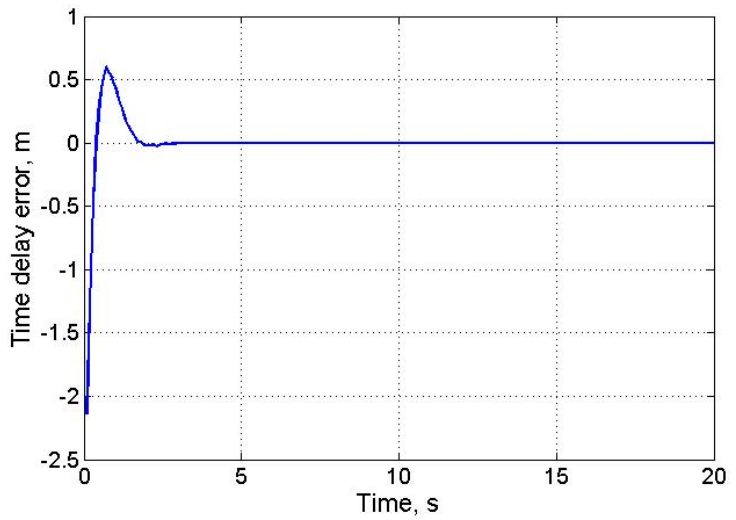

Fig. 4. A realization of a navigation signal delay tracking error

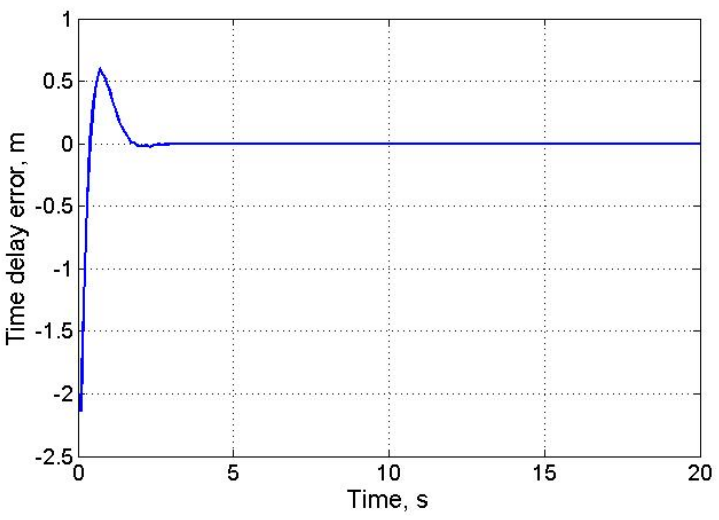

Fig. 6. A realization of a jamming signal delay tracking error

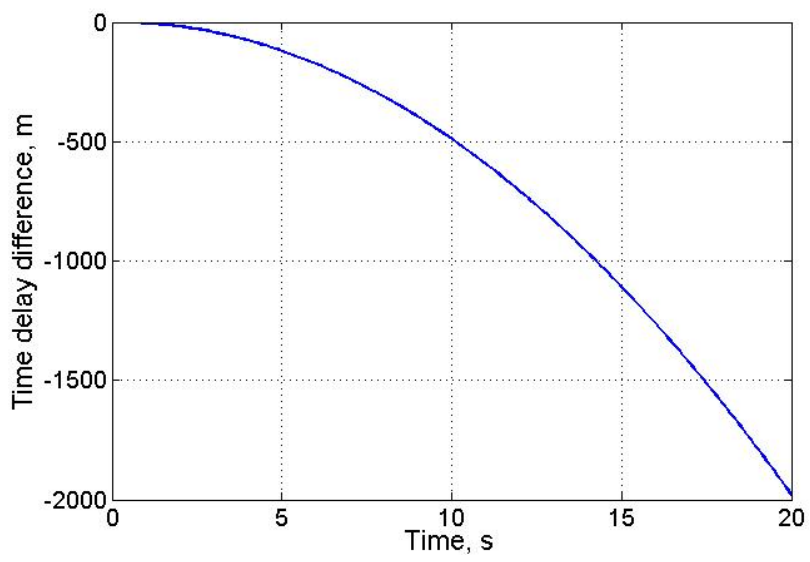

Fig. 7. A realization delay difference between navigation and jamming signals 
From Fig. 7 one can see that delay difference between navigation and jamming signals is changed considerably. From Figs. $3-7$ it is followed that in the synthesized optimal spacetime filtering algorithm there are implemented joint navigation signal tracking and jamming signal. Let's now consider space-time filtering systems in which there now used compensation the jamming signal in the navigation signal tracking system and compensation the navigation signal in the jamming signal tracking system (autonomous tracking systems). Appropriate plots are given in Figs. 8-11.

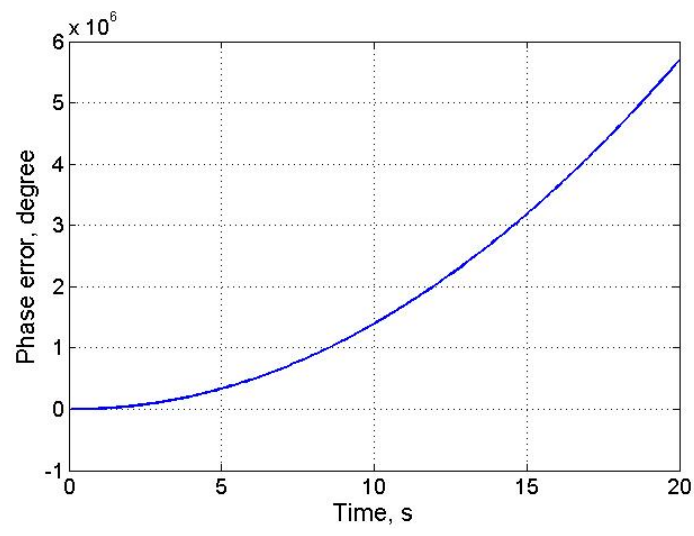

Fig. 8. A realization of a navigation signal phase tracking error in an autonomous tracking system

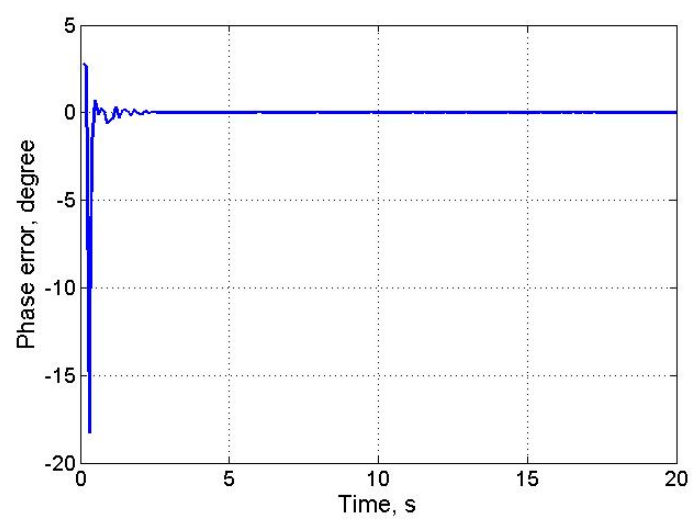

Fig. 10. A realization of a jammig signal phase tracking error in an autonomous tracking system

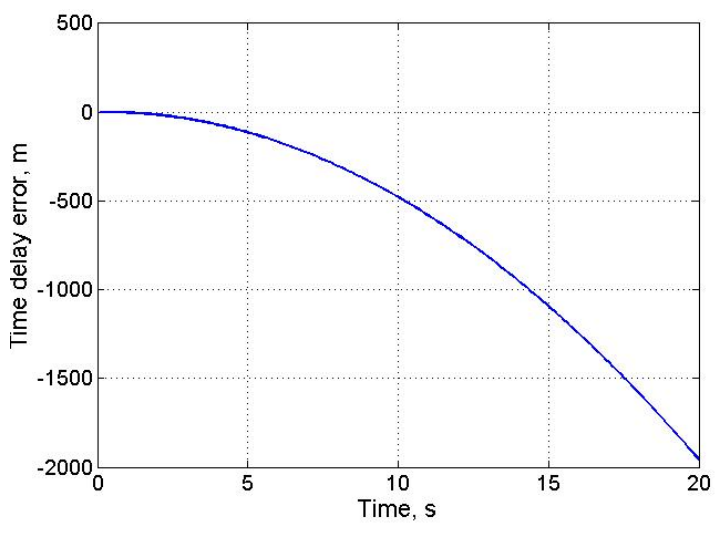

Fig. 9. A realization of a navigation signal dlay tracking error in an autonomous tracking system

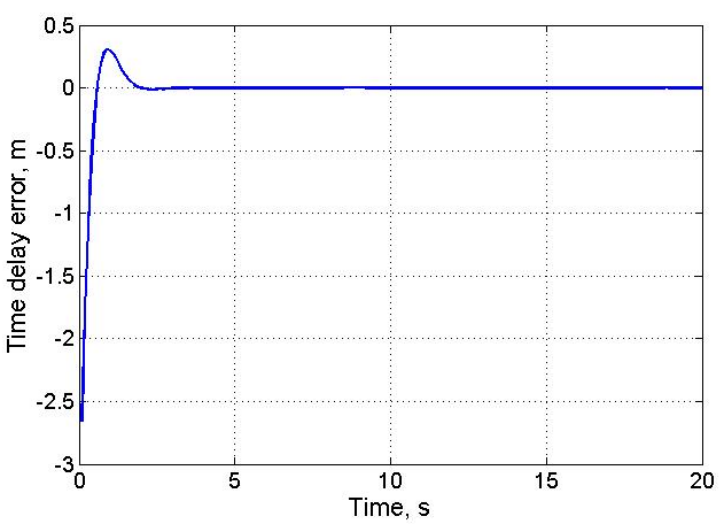

Fig. 11. A realization of a jamming signal delay tracking error in an autonomous tracking system

From Figs. 8-9 one can see that in this case navigation signal tracking is broken off. From Figs. 10-11 one can see that in this case jamming signal tracking is successfully passed.

Thereby on application of an autonomous navigation tracking system it is occurred tracking loss when an aimed spoofing jamming with more greater power relative to navigation signal power is acted. In the synthesized optimal space-time filtering system we have reliable navigation signal 
and jamming signal tracking.

\section{Conclusion}

In this article the algorithm of space-time processing received satellite navigation signal and spoofing jamming is synthesized using theory of information processes optimal filtering. In the synthesized algorithm in the navigation signal tracing system there are performed compensation of jamming signal action with a glance of its spatial and temporal characteristics. There is derived equations that are described the complex filtering system for analysis of regular processes transformation in the system. From held analysis it is followed that in the synthesized optimal space-time filtering system we have reliable navigation signal and jamming signal tracking when an aimed spoofing jamming has a little more power relative to navigation signal power.

\section{References}

[1] P.Misra, P.Enge, Global Positioning System., Measurements and Performance. Second edition, 2012.

[2] E.D.Kaplan, C.J.Hegarty, Understanding GPS principles and applications., Artech House Inc., Norwood, Massachusetts, 2006.

[3] V.N.Tyapkin, D.D.Dmitriev, T.G.Moshkina, The potential noise immunity of consumer navigation equipment satellite navigation systems., Vestnik SibGAU, (2012), no. 3, 113-119 (in Russian).

[4] S-J.Kim, R.A.Iltis, STAP for GPS Receiver Synchronization, IEEE Transaction on Aerospace and Electronic Systems, 40(2004), no. 1, 132-143.

[5] R.A.Monzigo, T.W.Miller, Introduction to Adaptive Arrays., John Wiley \& Sons, New York, 1980.

[6] S.G.Carlson, C.A.Popeck, M.H.Stockmaster, C.E.McDowell, Rockwell Collins Flexible Digital Anti-Jam Architecture., ION ION GPS/GNSS 2003, Portland, OR, 2003, 1843-1853.

[7] M.Sgammini, F.Antreich, L.Kurz, M.Meurer, T.G.Noll, Blind adaptive beamformer based on orthogonal projections for GNSS, ION GNSS 2012, Nashville, TN, 2012.

[8] S.N.Efimov, V.N.Tyapkin, D.D.Dmitriev, V.A.Terskov, Methods of assessing the characteristics of the multiprocessor computer system adaptation unit., Journal of SFU, Mathematics \& physics, $\mathbf{9}(2016)$, no. 3, 288-295.

[9] V.N.Tyapkin, Minimum variance distortionless response adaptive beamforming, Naukoemkie tekhnologii, $\mathbf{1 7}(2016)$, no. 8, 39-43 (in Russian).

[10] R.O.Schmidt, Multiple Emitter Location and Signal Parameter Estimation, IEEE Transactions on Antennas and Propagation, AP-34(1986), no. 3, 276-280.

[11] H.Krim, M.Viberg, Two Decades of Array Signal Processing Research, IEEE Signal Processing Magazine, 1996, 68-94. 
[12] A.I.Perov, V.N.Kharisov., GLONASS. Principles of construction and operation, Moscow, Radio, 4th ed., Revised, 2010 (in Russian).

[13] A.I.Perov, Statistical theory of radio systems, Radio Engineering, 2003 (in Russian).

\title{
Синтез оптимального алгоритма пространственно- временной обработки спутникового навигационного сигнала и сигнала имитационной помехи
}

\author{
Александр И. Перов \\ Сергей П. Ипполитов \\ Национальный исследовательский университет "МЭИ" \\ Красноказарменная, 14, Москва, 111250 \\ Россия
}

В статъе методами теории оптимальной фильтрации синтезирован оптимальный алгоритм пространственно-временной обработки спутникового навигационного сигнала и сигнала имитационной помехи. Приведены уравнения оптимальной пространственно-временной фильтрации и результаты анализа статистических характеристик такого алгоритма, иллюстрирующие его работоспособность и преимущества.

Ключевые слова: спутниковая навигачия, имитационная помеха, оптимальный алгоритм, слежение, пространственно-временная обработка. 